\title{
EXPLICIT ALGEBRAIC REYNOLDS STRESS MODELS FOR ANISOTROPIC WALL-BOUNDED FLOWS
}

\author{
F. R. Menter ${ }^{1}$, A. V. Garbaruk ${ }^{2}$, and Y. Egorov ${ }^{1}$ \\ ${ }^{1}$ ANSYS Germany GmbH \\ Germany \\ ${ }^{2} \mathrm{NTS}$
}

St. Petersburg, Russia

\begin{abstract}
In the present paper, two variants of Explicit Algebraic Reynolds Stress Model (EARSM) are presented and applied to a number of test cases. Both formulations start from the Wallin-Johansson (WJ) EARSM stress-strain relationship. The goal of the first step was to combine the EARSM with the $\omega$-equation-based Baseline (BSL) model, to avoid freestream sensitivities and ambiguities in comparison with the Shear Stress Transport (SST) model. This could be achieved by a slight change in the $A_{1}$ constant. In addition, the standard eddy-viscosity formulation is used in the diffusion terms of the $k$ - and the $\omega$-equations. Secondly, a simplified version of the stress-strain relationship was developed. It is based on a linear form of the implicit algebraic model. It is not clear at the time if this formulation possess significant advantages against the WJ stress-strain model. For the current cases, both variants produced essentially identical results. Several test cases have been computed. The main interest in the simulations was on corner flow separation.
\end{abstract}

\section{INTRODUCTION}

Current industrial and aeronautics computational fluid dynamics (CFD) simulations are largely based on linear eddy-viscosity turbulence models (LEVM). There are several reasons why higher order models like RSM have rarely made their way into mainstream industrial CFD applications, even though they are available in most general-purpose codes. The two main reasons are the increased computational requirements, often caused by a lack of robustness and the observation that such models, in many cases, did not result in a systematic improvement of results. Still there are numerous areas where EV models are known

This is an Open Access article distributed under the terms of the Creative Commons Attribution-Noncommercial License 3.0, which permits unrestricted use, distribution, and reproduction in any noncommercial medium, provided the original work is properly cited. 
to fail systematically and where further model improvements are required. The goal should be to allow the inclusion of specific additional effects without a large penalty on speed and robustness.

Explicit Algebraic RSM [1-4] offer an attractive framework for such enhancements. These models can be considered as a subset of nonlinear constitutive relations in which a part of the higher-order description of physical processes on the RSM-level is transferred into the two-equation modeling level. As a result, they are much less demanding than RSM from the computational standpoint and, at the same time, are capable of reproducing some important features of turbulence (e.g., its anisotropy in the normal stresses), which are beyond the capabilities of LEVM. The emphasis in the development of EARSM has largely been on the correct mathematical formulation of the stress-strain relationship and not so much in the formulation of an industrial CFD model (see, however, [5]).

When increasing the complexity in the formulation of a turbulence model, one should first answer the question: which types of flows will benefit from it? One of the weaknesses of EARSMs is that they do not naturally account for swirling and rotating flows. This being one of the major practical arguments for RSM, one has to find other areas where EARSM could be beneficial (or add appropriate rotation terms). One such area is the prediction of flows parallel to corners formed by intersecting walls, as observed in wing-body junctions or hub-blade regions. There is a strong indication that LEVM predict much too early separation from such corners when the flow is exposed to adverse pressure gradients. This can have a severe impact on the computed performance characteristics of these technical devices. The goal of the current paper is to explore model enhancements which allow the inclusion of such effects at the lowest level of complexity. For this purpose, two EARSM formulations will be investigated. The underlying idea being that the secondary flow of the second kind which is observed near corners is the main mechanism for obtaining delayed separation in these regions. In other words, it is anticipated that the secondary flow caused by the differences in the normal stresses drives additional momentum into the corner, thereby delaying separation. The goal is the identification of the most appropriate and simplest EARSM formulation which allows the inclusion of anisotropic effects into the formulation. There are two areas which will be investigated.

The first is the formulation of a scale-equation used in combination with the EARSM. Experience over the last decade has shown that $\omega$-equation-based models offer significant advantages over $\varepsilon$-equation-based models, especially if integration through the viscous sublayer is desired [6]. Unfortunately, the standard $\omega$-equation of Wilcox in its different forms is not suitable due to the persistent freestream sensitivity of this model, even in its latest version $[6,7]$. In addition, the SST model [7] has been widely used in aerodynamic and other industrial flow simulations. It is, therefore, desirable to formulate the EARSM on the basis of a scale-equation very similar to the SST model in order to isolate the impact of 
the stress-strain relationship on the solution. The starting point is, therefore, the BSL model, which underlies the SST model [7]. It will be shown that an EARSM can be formulated without a need for recalibrating the BSL model. This allows the direct comparison of the EARSM stress-strain relationship with the SST model formulation, without additional changes of other parts of the model.

The second area of interest is the formulation of the nonlinear stress-strain relationship. Here, numerous options are available in the literature and can be used as a starting point. Starting point in this paper is the stress-strain relation proposed by Wallin and Johansson [4, 8] which already provides a relatively simple EARSM closure. In addition, the question is posed: "what is the simplest EARSM which would still account for the anisotropy in the normal stresses in wall-bounded flows?" and would thereby allow the prediction of secondary flow into the corner. A model variant is explored which is linear in the implicit formulation, resulting in a simplification of the WJ stress-strain relation.

The models are tested for a set of flows with an emphasis of corner flow behavior. In addition, a flat plate boundary layer will be shown to demonstrate that the model gives the correct wall shear stress and logarithmic boundary layer profile. In some of the testcases, a comparison with the SST model is given. It should be emphasized that the superior performance for some of the corner flow cases does not imply a general recommendation for replacing the SST with the EARSM at current point. For such a recommendation, significantly more testing and model optimization (especially, in terms of robustness) is required.

\section{MODEL FORMULATION}

\subsection{The Wallin-Johansson Stress-Strain Relationship}

The starting point for the current investigation is the EARSM formulation of Wallin and Johansson [4]. The main contribution of WJ lies in a compact formulation of an EARSM based on a slightly simplified Launder-Reece-Rodi RSM. In the framework of this model, the anisotropy tensor, $a_{i j}$, defined through the Reynolds stress tensor $\tau_{i j}$

$$
\tau_{i j}=\overline{u_{i}^{\prime} u_{j}^{\prime}}=k\left(a_{i j}+\frac{2}{3} \delta_{i j}\right)
$$

is decomposed into a tensor basis as follows:

$$
a_{i j}=\beta_{1} T_{1, i j}+\beta_{2} T_{2, i j}+\beta_{3} T_{3, i j}+\beta_{4} T_{4, i j}+\beta_{6} T_{6, i j}+\beta_{9} T_{9, i j}
$$

where 


$$
\left.\begin{array}{c}
T_{1, i j}=S_{i j} ; \quad T_{2, i j}=S_{i k} S_{k j}-\frac{1}{3} I I_{S} \delta_{i j} ; \\
T_{3, i j}=\Omega_{i k} \Omega_{k j}-\frac{1}{3} I I_{\Omega} \delta_{i j} ; \quad T_{4, i j}=S_{i k} \Omega_{k j}-\Omega_{i k} S_{k j} ; \\
T_{6, i j}=S_{i k} \Omega_{k l} \Omega_{l j}+\Omega_{i k} \Omega_{k l} S_{l j}-\frac{2}{3} I V \delta_{i j}-I I_{\Omega} S_{i j} ; \\
T_{9, i j}=\Omega_{i k} S_{k l} \Omega_{l m} \Omega_{m j}-\Omega_{i k} \Omega_{k l} S_{l m} \Omega_{m j}+\frac{1}{2} I I_{\Omega}\left(S_{i k} \Omega_{k j}-\Omega_{i k} S_{k j}\right) .
\end{array}\right\}
$$

It should be noted that the tensor base for the EARSM has been modified from the one proposed by Wallin and Johansson [4] in order to separate the two- (2D) and three-dimensional (3D) contributions. This regrouping does not change the model formulation, but does formally result in a different set of coefficients $\beta_{i}$. The quantities $S_{i j}$ and $\Omega_{i j}$ in (3) are the nondimensional strain and vorticity tensors of the mean flow:

$$
S_{i j}=\frac{\tau}{2}\left(\frac{\partial U_{i}}{\partial x_{j}}+\frac{\partial U_{j}}{\partial x_{i}}\right) ; \quad \Omega_{i j}=\frac{\tau}{2}\left(\frac{\partial U_{i}}{\partial x_{j}}-\frac{\partial U_{j}}{\partial x_{i}}\right)
$$

where $\tau$ is the time scale with a Kolmogorov limiter [9]:

$$
\tau=\max \left(\frac{1}{C_{\mu} \omega}, 6 \sqrt{\frac{\nu}{C_{\mu} k \omega}}\right)
$$

and the tensor invariants $I I_{S}, I I_{\Omega}$ and $I V$ read as

$$
I I_{S}=S_{i j} S_{j i} ; \quad I I_{\Omega}=\Omega_{i j} \Omega_{j i} ; \quad I V=S_{i k} \Omega_{k j} \Omega_{j i} .
$$

The coefficients of the tensor basis $\beta_{i}$ in (2) are defined as:

$$
\beta_{1}=-\frac{N}{Q} ; \quad \beta_{2}=0 ; \quad \beta_{3}=-\frac{2 I V}{N Q_{1}} ; \quad \beta_{4}=-\frac{N}{Q} ; \quad \beta_{6}=-\frac{N}{Q_{1}} ; \quad \beta_{9}=\frac{1}{Q_{1}}
$$

with

$$
Q=\frac{N^{2}-2 I I_{\Omega}}{A_{1}} ; \quad Q_{1}=\frac{Q}{6}\left(2 N^{2}-I I_{\Omega}\right)
$$

where

$$
N=C_{1}^{\prime}+\frac{9}{4} \frac{P_{k}}{\varepsilon}
$$

$A_{1}=1.2$, and $C_{1}^{\prime}=(9 / 4)\left(C_{1}-1\right), C_{1}=1.8$. Here, $N$ is the solution of the cubic equation:

$$
N^{3}-C_{1}^{\prime} N^{2}-\left(2.7 I I_{S}+2 I I_{\Omega}\right) N+2 C_{1}^{\prime} I I_{\Omega}=0
$$

which is given by (the $2 \mathrm{D}$ version of the $N$-equation formulation was selected): 


$$
N= \begin{cases}\frac{C_{1}^{\prime}}{3}+\left(P_{1}+\sqrt{P_{2}}\right)^{1 / 3}+\operatorname{sign}\left(P_{1}-\sqrt{P_{2}}\right)\left|P_{1}-\sqrt{P_{2}}\right|^{1 / 3} & \text { at } P_{2} \geq 0 \\ \frac{C_{1}^{\prime}}{3}+2\left(P_{1}^{2}-P_{2}\right)^{1 / 6} \cos \left(\frac{1}{3} \arccos \left(\frac{P_{1}}{\sqrt{P_{1}^{2}-P_{2}}}\right)\right) & \text { at } P_{2}<0\end{cases}
$$

with

$$
P_{1}=C_{1}^{\prime}\left(\frac{C_{1}^{\prime 2}}{27}+\frac{9}{20} I I_{S}-\frac{2}{3} I I_{\Omega}\right) ; \quad P_{2}=P_{1}^{2}-\left(\frac{C_{1}^{\prime 2}}{9}+\frac{9}{10} I I_{S}+\frac{2}{3} I I_{\Omega}\right)^{3} .
$$

Note that the model does not include the term $T_{9, i j}\left(\beta_{0}=0\right)$ : as was shown by the preliminary computations, this term does not cause any visible alteration of the obtained solution.

It should also be mentioned that no attempt was made to account for the near-wall anisotropy of turbulence in the buffer region below the logarithmic layer. Enhancements for this purpose have been tested and found to have very little impact on the solution, but strongly reduce robustness.

\subsection{Baseline-Explicit Algebraic Reynolds Stress Models}

The WJ stress-strain relationship above was originally combined with the $k-\omega$ equations as given by Wilcox [4]. In order to avoid the freestream sensitivity of the Wilcox model, the WJ stress-strain relation is combined here with the BSL $k-\omega$ model [7]. To avoid a recalibration or the need for additional terms in the BSL model, a slight recalibration of the WJ-EARSM was performed, namely a 4 percent increase of the $A_{1}=1.245$ parameter (instead of $A_{1}=1.2$ in WJ equations (4), (5) [10]. This allows matching the logarithmic wall layer without changing the EARSM stress-strain relationship. It is also important to point out that the eddy-viscosity used in the diffusion terms of the $k$-and $\omega$-equations is computed from $\nu_{t}=k / \omega$. This choice avoids the problems observed and resolved by Hellsten and Laine [5] near the boundary layer edge.

The complete BSL-EARSM reads:

$$
\begin{aligned}
& \frac{D k}{D t}=\nabla\left(\left(\nu+\delta_{k} \nu_{t}\right) \nabla k\right)+\tilde{P}_{k}-\beta^{*} k \omega \\
& \frac{D \omega}{D t}=\nabla\left(\left(\nu+\delta_{\omega} \nu_{t}\right) \nabla \omega\right)+\frac{\gamma \omega}{k} \tilde{P}_{k}-\beta \omega^{2}+\frac{\delta_{d}}{\omega}(\nabla k)(\nabla \omega)
\end{aligned}
$$

where $P_{k}$ is given by (using a production limiter as in the SST model):

$$
\tilde{P}_{k}=\min \left(-\tau_{i j} \frac{\partial U_{i}}{\partial x_{j}}, 10 \rho \beta^{*} k \omega\right) .
$$


The model constants are defined via the corresponding constants of the $k-\varepsilon$ (subscript "1") and $k-\omega$ (subscript "2") branches of the BSL model with the use of the blending function $F_{1}$ :

$$
\begin{gathered}
\sigma_{k}=F_{1} \sigma_{k 1}+\left(1-F_{1}\right) \sigma_{k 2} ; \quad \sigma_{\omega}=F_{1} \sigma_{\omega 1}+\left(1-F_{1}\right) \sigma_{\omega 2} ; \\
\beta=F_{1} \hat{\beta}_{1}+\left(1-F_{1}\right) \hat{\beta}_{2} ; \quad \sigma_{d}=2\left(1-F_{1}\right) \sigma_{\omega 2} ; \\
\sigma_{k 1}=0.5 ; \sigma_{k 2}=1.0 ; \sigma_{\omega 1}=0.5 ; \sigma_{\omega 2}=0.856 ; \hat{\beta}_{1}-0.075 ; \hat{\beta}_{2}=0.0828 ; \\
\beta^{*}=C_{\mu}=0.09 ; \quad \gamma=\frac{\beta}{\beta^{*}}-\frac{\sigma_{\omega} \kappa^{2}}{\sqrt{\beta^{*}}}
\end{gathered}
$$

where the function $F_{1}$ reads as ( $d$ is the distance to the nearest wall)

$$
F_{1}=\tanh \left(\arg _{1}^{4}\right) ; \arg _{1}=\min \left[\max \left(\frac{\sqrt{k}}{C_{\mu} \omega d}, \frac{500 \nu}{\omega d^{2}}\right) \frac{2 k \omega}{d^{2}(\nabla k)(\nabla \omega)}\right] .
$$

\subsection{Simplified Baseline-Explicit Algebraic Reynolds Stress Models}

In order to explore the most simplified EARSM formulation which would still allow the inclusion of near-wall normal stress anisotropies, the formulation of WJ was simplified in the nonlinear portion of the model. The nonlinearity in the implicit ARSM results from the production term $P_{k}$, which again contains the unknown Reynolds Stresses (or anisotropies). A simplified formulation can be obtained by replacing the ratio of production/dissipation in (5) by the following equilibrium assumption (similar to the SST assumption):

$$
\frac{P_{k}}{\varepsilon}=\sqrt{2 C_{\mu} I I_{S}}
$$

This results in a linear formulation for the quantity $N$ instead of (6)-(8):

$$
N=C_{1}^{\prime}+\frac{9}{4} \frac{P_{k}}{\varepsilon}=C_{1}^{\prime}+\frac{9}{4} \sqrt{2 C_{\mu} I I_{S}} .
$$

The rest of the model formulation remains unchanged. The hope of this simplification is to improve the robustness of the model by reducing its nonlinearities. This is intended as a test of model variants. It is clear that generality is sacrificed by this simplification, but for the goal of computing corner separation in wall boundary layer flows, the assumption seems justified.

The rationale behind Eq. (9) is based on the observation that in equilibrium boundary (mixing) layers, the (SST) relationship holds:

$$
-\overline{u \nu}=\sqrt{c_{\mu}} k
$$

where $-\overline{u \nu}$ is the principle shear stress. As a result, one obtains: 


$$
P_{k}=-u \nu \frac{d U}{d y}=\sqrt{c_{\mu}} k \frac{d U}{d y}
$$

and with

$$
\left|\frac{d U}{d y}\right| \rightarrow \sqrt{2 I I_{S} c_{\mu}^{2} \omega^{2}}
$$

follows Eq. (9).

\section{FLOW SOLVER}

The above models have been implemented into a prototype version of the ANSYS-CFX-12 solver and an in-house code of NTS [11]. Cross-validation of the codes with successive grid refinement for all testcases has shown that the model implementation is code independent.

\section{TEST CASES}

\subsection{Flat Plate Boundary Layer}

The first tests were carried out for a flat plate boundary layer with zero pressure gradient. The simulations are compared against experimental data of Wieghart and Tillmann [12]. Three meshes were used in the tests to ensure grid independence. The grids are all of resolution with $y^{+}<1$. Figure 1 shows the wall

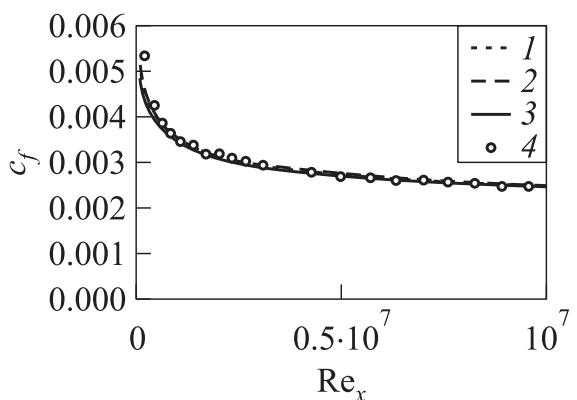

(a)

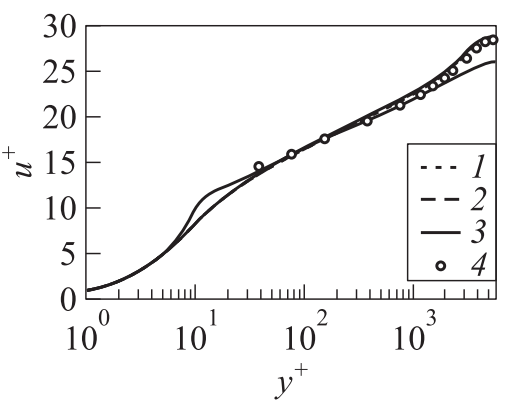

(b)

Figure 1 Comparison of skin-friction coefficient distributions $(a)$ and velocity profiles $(b)\left(\operatorname{Re}_{x}=9.37 \cdot 10^{6}\right)$ in a flat plate boundary layer computed with the use of different EARSM (1 - BSL-EARSM and 2 - S-BSL-EARSM) and SST (3) models with experimental data of [12] (4) 
shear stress distribution $c_{f}$ and the velocity profile in a logarithmic scale. For both plots, the new models (BSL-EARSM and Simplified BSL-EARSM (S-BSLEARSM)) agree well with the data.

\subsection{Three-Dimensional Developed Flow in a Square Duct}

This testcase represents an example of flows which show an essential influence of the anisotropy of turbulence on the mean flow. In particular, the anisotropy causes the formation of a secondary flow, which is beyond the capabilities of LEVM.

The computations were performed at a Reynolds number based on the mean friction-velocity and the channel half-width, $h$, equal to 6000 which corresponds to the conditions of the direct numerical simulation (DNS) of Huser and Biringen [13].

The computations are carried out in $\mathrm{a}^{1 / 4}$ quadrant of the whole channel with symmetry conditions imposed at $y=z=h$ (Fig. 2). At the streamwise boundaries, a periodic condition was applied and at the solid walls, no-slip conditions were used. The grid in the $Y Z$-plane also shown in the figure has a size of $51 \times 51$. It is refined near the solid wall so that the near-wall values of $y^{+}$and $z^{+}$are not higher than 0.3 .

Figure 3 shows the axial $u$-velocity and the $v$-velocity normal to the lower wall along a line $y=z$. Both EARSM formulations give essentially the same result.

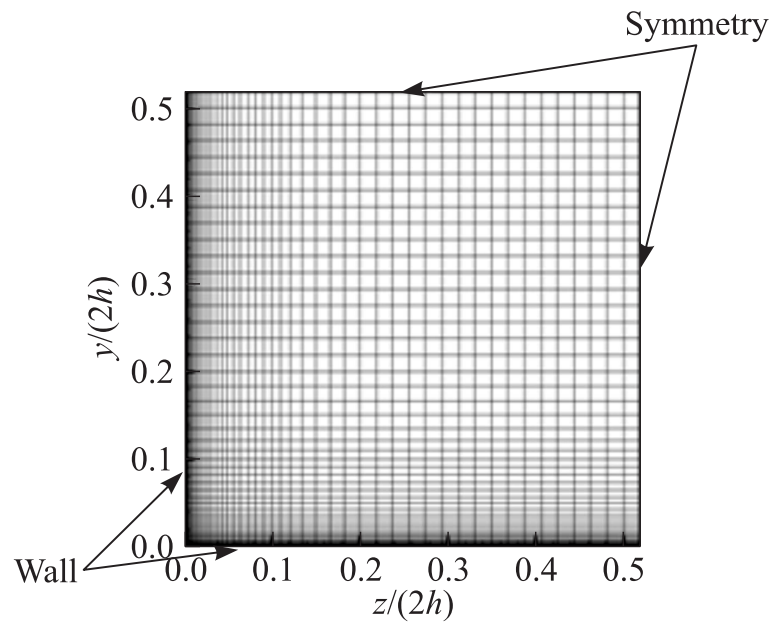

Figure 2 Computational domain and mesh used for computations of the fully developed square duct case 


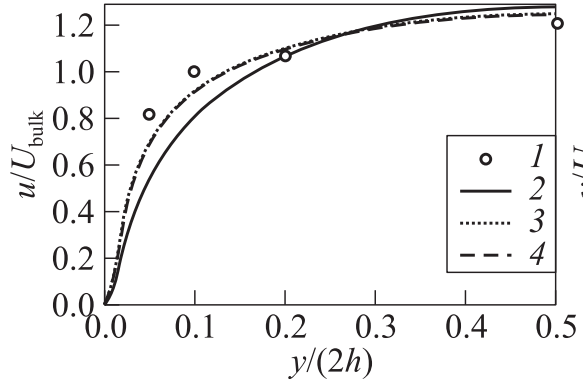

(a)

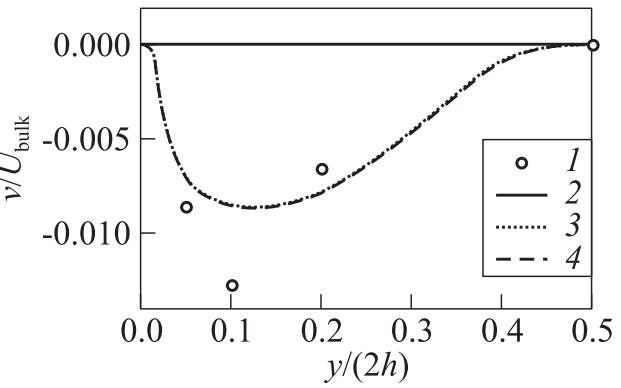

(b)

Figure 3 Comparison of mean and diametric velocity along line $y=z: 1-$ DNS; 2 - SST; 3 - BSL-EARSM; and 4 - S-BSL-EARSM

The SST model, as all LEVMs, predicts a zero value for the $v$-velocity, meaning that no secondary flow is produced in the $y-z$ plane. This is a known deficiency of LEVMs, as the secondary flow is driven by differences in the normal stresses, which cannot be accounted for by LEVMs. Figure $3 a$ shows the main effect of interest in the current study, namely, the increased axial momentum transfer into the corner, manifesting itself by a higher axial velocity near the corner walls. It is expected that this effect will help to delay corner separation under adverse pressure gradients. The current comparison is done for a relatively low Reynolds number. Further tests at higher Reynolds numbers are still required.

\subsection{Incompressible Flow in a Rectangular Diffuser}

This flow studied experimentally by Cherry et al. [14] is a much more challenging testcase than the developed flow considered above. Along with the turbulence anisotropy typical of rectangular channel flows, it involves an adverse pressure gradient causing separation. This separation has proven very sensitive to details of turbulence modeling [15]. It seems clear that the anisotropy of the normal stresses has to be accounted for in order to avoid the formation of an incorrect flow topology.

The geometry of the diffuser and coordinate system used in the computations are shown in Fig. 4. The origin of the coordinate system in the $x$-direction is located at the cross section of the diffuser corresponding to the intersection of its straight inlet and the inclined walls.

According to the experimental setup, the flow at the diffuser inlet is treated as a fully developed flow in a rectangular duct with the bulk velocity of $1 \mathrm{~m} / \mathrm{s}$. Therefore, the velocity in the inlet plane has to be specified based on the precursor computation of the developed duct flow with the use of corresponding (the same as that used for the diffuser computation) turbulence model. 

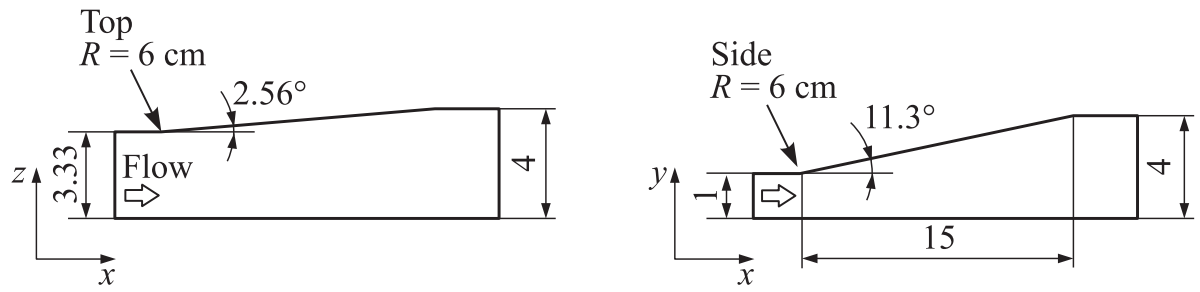

Figure 4 Geometry of the rectangular diffuser. Dimensions are in millimeters
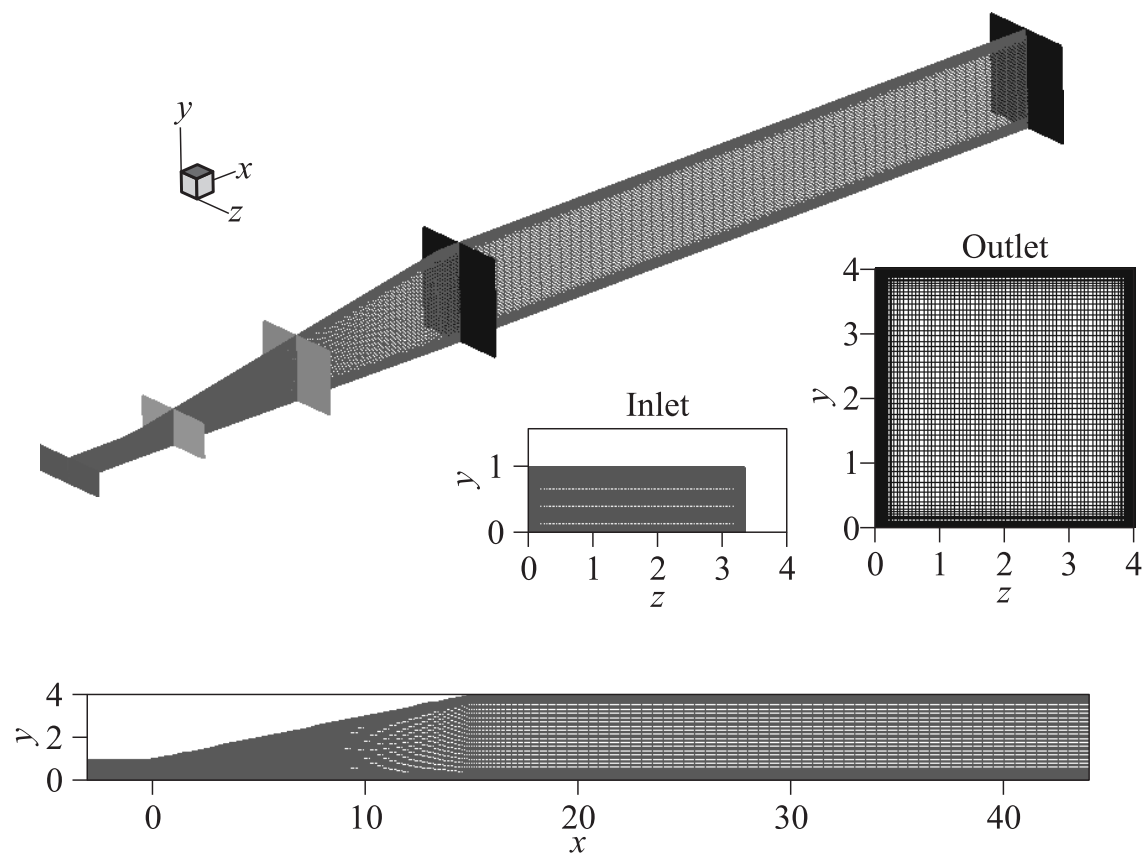

Figure 5 Computational domain and grid used for the rectangular diffuser flow case

Figure 5 presents the computational domain and the mesh used in the computations. It has $145 \times 91 \times 121$ nodes in the $x, y$, and $z$ directions, respectively, and is refined in $y$ - and $z$-directions near all the walls (the maximum value of $y^{+}$ is 0.7 ) and, in the $x$-direction, inside the diffuser. The grid for the simulation of the inlet section consists of $91 \times 121$ nodes.

Figure 6 shows a comparison of the velocity field in the streamwise plane $x=16 \mathrm{~cm}$ against experimental data. In order to investigate the influence of the anisotropy on the flow, the BSL-EARSM was also run with only the isotropic 


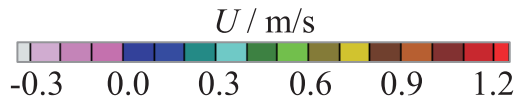

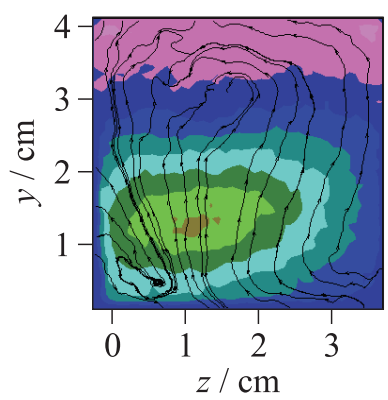

(a)

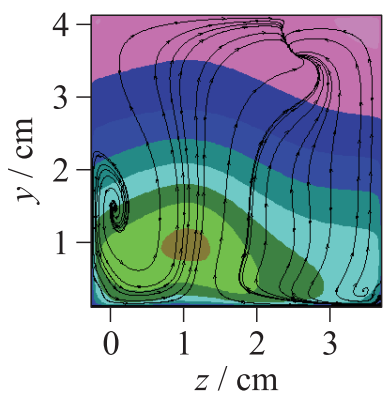

(b)

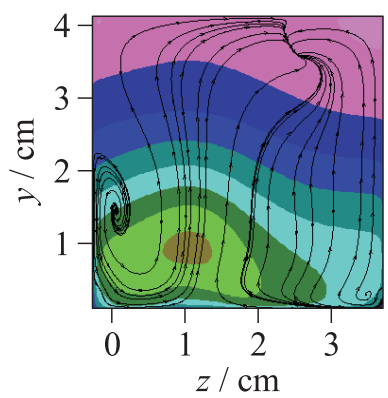

(c)

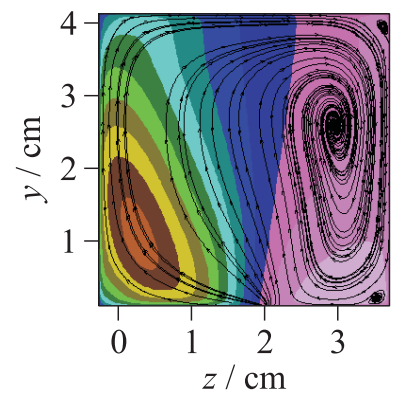

(d)

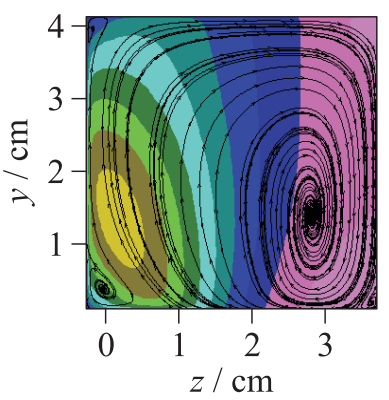

(e)

Figure 6 Comparison of velocity fields at section $x=16 \mathrm{~cm}$ : $(a)$ experiment; (b) BSL-EARSM; (c) S-BSL-EARSM; $(d)$ SST; and $(e)$ linear part of EARSM. (Refer Menter et al., p. 99.)

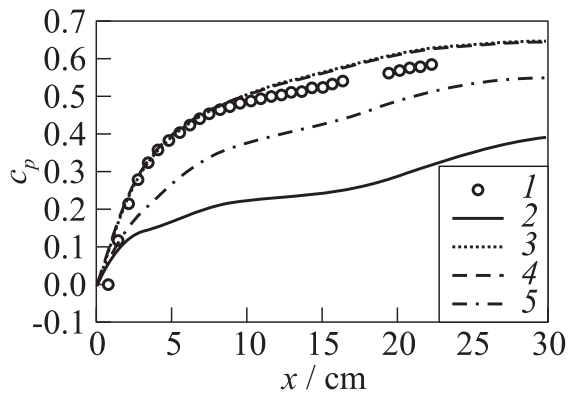

Figure 7 Comparison of pressure coefficient for 3D diffuser: 1 - experiment; $2-$ SST; 3 - BSL-EARSM; 4 - S-BSL-EARSM; and 5 - linear part of EARSM 
contribution $\beta_{1} T_{1}$ (see Eqs. (1)-(3)) activated. It can be seen that the isotropic contribution of the EARSM and the SST model are not able to predict the correct flow topology. This is the main argument for using anisotropic formulations for such flows.

Figure 7 shows the corresponding wall pressure distributions. Again, the linear models produce incorrect results, reflecting their problems in representing the flow topology properly.

\subsection{Transonic Flow Past DLR F-6 Airplane Configuration}

This test case was first calculated with the SST model using ANSYS-CFX for the 2nd AIAA Drag Prediction Workshop [16, 17]. The same simulation setup was used here to compare the S-BSL-EARSM and its isotropic version (activating only $\beta_{1}$ ) with the SST model. The variant for the geometry with mounted engine, as shown in Fig. 8, was selected for comparison, with an angle of attack of $1^{\circ}$, providing a lift coefficient of about 0.5. This variant was also used by Peng and Eliasson [18] to compare the size of separation zones at the 3D wing-fuselage corner (upper wing surface) and behind the engine (lower wing surface) between the results of several different turbulence models, including SST and EARSM (see also paragraph 20.3.3 in [19]). In Fig. 8, these areas of interest are marked by circles.

Transonic flow calculations at a Mach number of 0.75 and $\operatorname{Re}=3 \cdot 10^{6}$ were performed on a block-structured grid of 8.4 million elements. References to the measurement carried out at ONERA are available in [16]. In order to evaluate the pure effect of anisotropy of the diagonal Reynolds stresses, one additional simulation was done including only the linear contribution $\beta_{1} T_{1}$ (see Eqs. (1)-

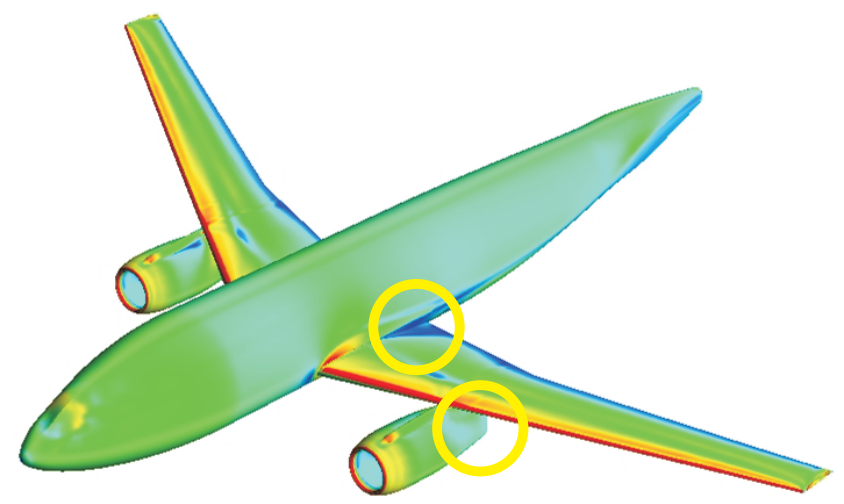

Figure 8 Geometry of DLR F6 wing-body-pylon-nacelle testcase. (Refer Menter et al., p. 100.) 

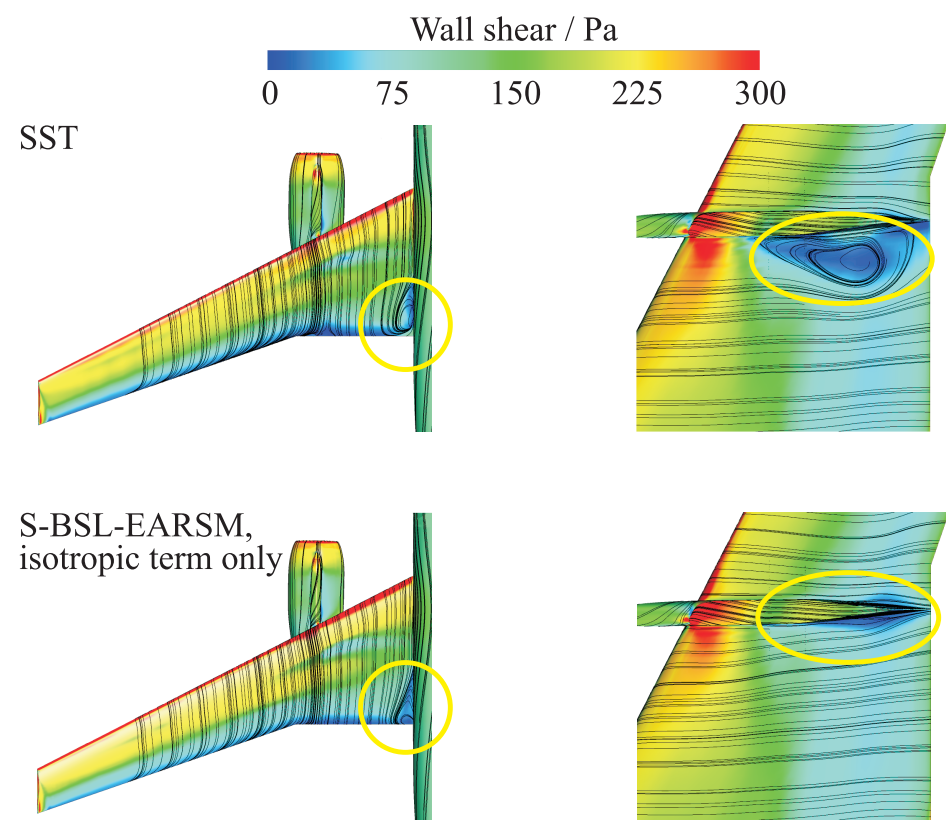

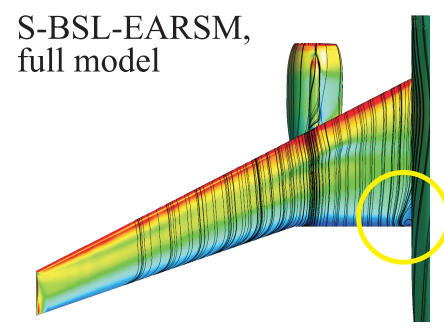

(a)

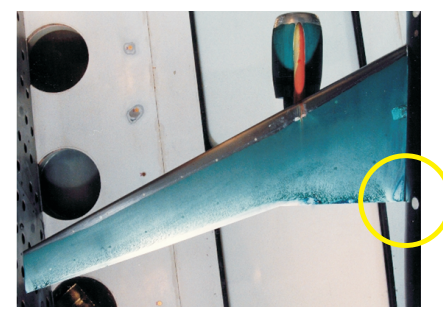

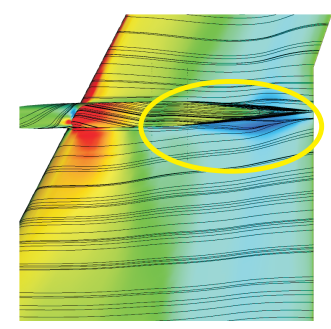

(b)

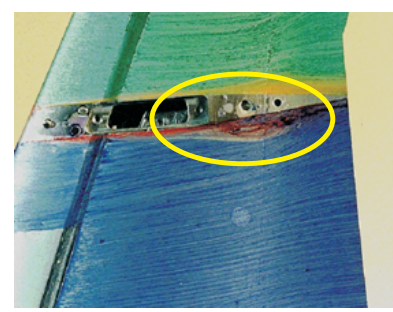

(c)

Figure 9 Separation zone at the wing-fuselage junction $(a)$ and behind the engine $(b)$; (c) oil film visualization in the experiment. (Refer Menter et al., p. 101.) 
(3)), i. e., using the isotropic part of the model. This is done, as the differences between the $\beta_{1}$ contribution and the SST models eddy-viscosity could also change some of the characteristics of the flow in the corner, without any effect of the stress anisotropy. The effect of the anisotropy can, therefore, best be seen by comparing the S-BSL-EARSM and the $\beta_{1}$-limited variant (it is to be emphasized that the $\beta_{1}$-limited variant is by no means recommended - it is only run to evaluate the impact of the anisotropic part of the EARSM formulation).

As already noticed with the earlier results, the SST model tends to overpredict the size of the corner separation zones, as shown in Fig. 9. The SBSL-EARSM model improves the result for the wing-fuselage corner separation on the upper wing surface (Fig. 9a). It is to be noted, however, that already the isotropic version ( $\beta_{1}$-limited variant) reduces the separation zone somewhat relative to SST. Therefore, not the entire effect is a result of the stress anisotropy. Nevertheless, the anisotropic model again significantly improves the solution in this region, resulting in a much improved comparison with the experimental oil flow picture.

The other corner separation zone sits on the lower wing surface behind the engine. It is also overpredicted by the SST model. However, it has fully disappeared in both the S-BSL-EARSM and the $\beta_{1}$-limited EARSM simulations (Fig. $9 b$ ). This change cannot, therefore, be attributed to the anisotropic representation of the stress-tensor alone. This model behavior will have to be further investigated and is likely a result of elliptic effects changing the flow on a more global level.

For this complex application, a reduced robustness of the solver was observed. The time step had to be reduced significantly, resulting in corresponding increase in computing times. This behavior of the model has, however, not been investigated systematically and needs further investigation.

\section{SUMMARY}

In the present paper, two variants of EARSM have been presented and applied to a number of testcases. Both formulations start from the WJ EARSM stressstrain relationship. The goal of the first step was to combine the EARSM with the $\omega$-equation based BSL model, to avoid freestream sensitivities and ambiguities in comparison with the SST model. This could be achieved by a slight change in the $A_{1}$ constant. In addition, the standard eddy-viscosity formulation is used in the diffusion terms of the $k$ - and the $\omega$-equations.

Secondly, a simplified version of the stress-strain relationship was developed. It is based on a linear form of the implicit algebraic model. It is not clear at the time if this formulation possesses significant advantages against the WJ stressstrain model. For the current cases, both variants produced essentially identical results. 
Several testcases have been computed. The flat plate confirms that the model produces correct wall shear stress levels and a proper logarithmic layer. The main interest in the simulations was on corner flow separation. The computation of the 3D Stanford diffuser shows that the inclusion of the stress anisotropy leads to a drastic improvement of the results for this case. The flow topology matches much better the experimentally observed flow and the wall pressure distribution improves significantly. It should, however, be emphasized that this is a very sensitive flow and that such drastic changes are not to be expected for most engineering applications. Finally, the flow around a wing-body-pylonnacelle configuration was simulated. The goal was again a reduction of the corner flow separation. On the upper wing-body intersection, a marked improvement attributed to the anisotropy was observed. On the lower wing-pylon intersection, the results are inconclusive, as they change already when using the isotropic form of the EARSM.

The current tests should be considered as a first iteration in the formulation of improved Reynolds-averaged Navier-Stokes models for flows with corner separation. Especially numerical robustness and the near wall formulation of the anisotropies will require further optimization. Nevertheless, the results are encouraging and demonstrate the potential of EARSM for such flows.

\section{ACKNOWLEDGMENTS}

The authors want to thank Stefan Wallin for his comments during the model formulation. This work was supported by the EU through the project ATAAC (Advanced Turbulence Simulation for Aerodynamic Application Challenges).

\section{REFERENCES}

1. Pope, S. B. 1975. A more general effective-viscosity hypothesis. J. Fluid Mech. 72:331-40.

2. Rodi, W. 1976. A new algebraic relation for calculating the Reynolds stresses. Ed. Z. Angew. Math. Mech. 56:219-21.

3. Gatski, T.B., and C.G. Speziale. 1993. On explicit algebraic stress models for complex turbulent flows. J. Fluid Mech. 254:59-78.

4. Wallin, S., and A. V. Johansson. 2000. An explicit algebraic Reynolds stress model for incompressible and compressible turbulent flows. J. Fluid Mech. 403:89-132.

5. Hellsten, A., and S. Laine. 2000. Explicit algebraic Reynolds stress modelling in decelerating and separating flows. AIAA Paper No. 2000-2313.

6. Menter, F. R. 2009. Review of the shear-stress transport turbulence model experience from an industrial perspective. Int. J. Comput. Fluid Dyn. 23(4):3005-316. 
7. Menter, F. R. 1994. Two-equation eddy-viscosity turbulence models for engineering applications. AIAA J. 32(8):1598-605.

8. Wallin, S., and A. V. Johansson. 1997. A new explicit algebraic Reynolds stress turbulence model for 3D flow. 11th Symposium on Turbulent Shear Flows Proceedings. Grenoble, France. 2:13.

9. Durbin, P. A. 1991. Near-wall turbulence closure modeling without "damping functions." Theor. Comput. Fluid Dyn. 3(1):1-13.

10. Wallin, S. 2009. Personal communication.

11. Strelets, M. 2001. Detached-eddy simulation of massively separated flows. AIAA Paper No. 2001-0879.

12. Wieghardt, K., and W. Tillmann. 1951. On the turbulent friction layer for rising pressure. NACA TM 1314.

13. Huser, A., and S. Biringen. 1993. Direct numerical simulation of turbulent flow in a square duct. J. Fluid Mech. 257:65-95.

14. Cherry, E. M., C. J. Elkins, and J. K. Eaton. 2007. Geometric sensitivity of 3-D separated flows. 5th Symposium (International)on Turbulence and Shear Flow Phenomena - TSFP5 Proceedings. Munich.

15. ERCOFTAC/IAHR. 2008. 13th Workshop on Refined Turbulence Modelling. http:// 130.83.243.201/ercoftac-sig15/workshop2008.html.

16. DPW. 2003. 2nd AIAA CFD Drag Prediction Workshop. http://aaac.larc. nasa.gov/tsab/cfdlarc/aiaa-dpw/. Orlando, FL.

17. Langtry, R. B., M. Kuntz, and F. R. Menter. 2004. Drag prediction of engineairframe interference effects with CFX-5. AIAA Paper No. 2004-0391.

18. Peng, S., and P. Eliasson. 2004. A comparison of turbulence models in prediction of flow around the DLR-F6 aircraft configuration. AIAA Paper No. 2004-4718.

19. Haase, W., B. Aupoix, U. Bunge, and D. Schwamborn, eds. 2006. FLOMANIA "A European initiative on flow physics modeling - results of the European-Union funded project 2002-2004." Notes on Numerical Fluid Mechanics and Multidisciplinary Design. Berlin/Heidelberg: Springer. 94. 